International Journal of Engineering \& Technology, $7(4)(2018) 2528-2532$
International Journal of Engineering \& Technology
SPC
Website: www.sciencepubco.com/index.php/IJET
doi: $10.14419 /$ ijet.v7it.14216
Research paper

\title{
ChestXthon: An algorithm for Abnormality Detection in Chest Radiographs
}

\author{
N.Sarada ${ }^{1}$, K Thirupathi Rao $^{2}$, K.V. Ramana $^{3}$ \\ ${ }^{1}$ Research Scholar Dept. of CSE, K L E F, Guntur \\ ${ }^{2}$ Professor, Dept. of CSE, KLE F, Guntur \\ ${ }^{3}$ Professor, Dept. of CSE, JNTUK, Kakinada \\ *Corresponding author E-mail:saradabusi@gmail.com
}

\begin{abstract}
Chest illnesses like heart failure, lung tumor or lung tuberculosis, and so on is frequently in view of chest X-ray images (CXR). The ailments are treatable on the off chance that they are recognized in their beginning times. Analyzing CXR is a tedious procedure. Now and again, therapeutic specialists had ignored the illnesses in their first examinations on CXR, and when the pictures were reevaluated, the malady signs could be detected. Furthermore, the quantity of CXR to look at is various and a long ways past the capacity of accessible therapeutic staff, particularly in creating nations. A PC supported finding (CAD) framework can check presumed zones on CXR for cautious examination by restorative specialists, and can give caution in the cases that need critical consideration. This paper reports our persistent work on developing an algorithm that aids the radiologists for the diagnosis of chest radiographs.
\end{abstract}

\section{Introduction}

Computer Aided Diagnosis

CAD (Computer Aided Diagnosis) are structures which are utilized for helping specialists for deciphering restorative pictures [1]. A portion of the imaging modalities are X-ray, MRI, and ultrasound diagnostics which are utilized as idealistic data for radiologists, Doctors and some logical expert for research are looking at them extensively in ideal day and age .It likewise has capacity predetermination utilizes as a part of computerized pathology within the sight of machine learning calculations and entire slide imaging. It is an integrative age which joins distinctive conditions of PC vision and manufactured knowledge with pathology and radiological picture handling. This appears to indicate that Computer Aided Diagnosis is starting to be as an application commonly in differential analysis and detection of many absolute styles of deformities in scientific pictures received in many evaluations via usage of different imaging modalities. In fact, CAD has grown to be the important research oriented topics in diagnostic radiology and medical imaging.

With Computer Aided Diagnosis (CAD), the performance of computers does no longer need to be corresponding to or higher than that with the aid of doctors, but wishes to be equivalent to that by way of doctors (synergy). It is being utilized in experimental circumstances for more than 40 years, it usually does now not replace the medical doctor or different skillful, but performs a role which is supporting to them.

"Interpreting a chest radiograph is to a great degree testing. Superimposed anatomical structures make the picture confused. Indeed, even experienced radiologists experience difficulty recognizing penetrates from the ordinary example of fanning veins in the lung fields, or identifying unobtrusive knobs that show lung growth. At the point when radiologists rate the seriousness of strange discoveries, huge interobserver and considerably intraobserver contrasts happen [2]. The clinical significance of chest radiographs, joined with their muddled nature, discloses the enthusiasm to create PC calculations to help radiologists in perusing chest diagnosis. The motivation behind this study is to order and quickly audit the writing on PC investigation of chest radiographs with an accentuation on the methods that have been utilized and on the errands these systems should explain.

\subsection{Objectives of the CAD system}

Computer Aided Diagnosis can be used for detection of "abnormalities" in chest $\mathrm{x}$ rays. Computer-aided diagnosis (CAD) allows image analysis by comparing images thereby reducing diagnostic time and enabling the Radiologist to analyse data with more detail. This system performs an analysis that uses a large set of texture features to compare each pixel in the image. Images can be scanned to high-resolution whole slides, which are decomposed into constituent scales. Each scale has a number of image features extracted. Each feature in the set is assessed for its performance and subsequently used to discriminate between "normal" and "abnormal" [3].This same principle can be applied to the detection of other (or any) radiological lesions or even certain histological features. The validation of the features in the set is time- and resource-intensive and requires constant qualitative assessment by an experienced radiologist.

\subsection{Application of CAD system}

There are different type of Computer Aided Diagnosis application such as breast, lung, and colon cancer. In Colon Cancer Computer Aided Diagnosis is available for revelation of colorectal polyps 
inside the colon in CT colonography. Polyps are little advancements that get up from the inner covering of the colon. Computer Aided Diagnosis recognizes the polyps through figuring of their trademark "knock like" shape [4]. To evade immoderate fake positives, It disregard the everyday colon divider, which includes the haustra folds .Computer Aided Diagnosis is able to stumble on polyps "neglected" with the aid of radiotherapist. In Breast Cancer Computer Aided Diagnosis is utilized as a part of screening mammography (X-shaft examination of the female chest). Screening mammography is used for the early revelation of chest disease. PC Aided Diagnosis systems are oftentimes connected to help portray a tumor as unsafe or liberal. It is extraordinarily snared in US and the Netherlands and is utilized as a part of expansion to human assessment, normally by using a radiologist. The primary Computer Aided Diagnosis framework for mammography became grow in a research assignment at the University of Chicago [5].Computer Aided Diagnosis frameworks have a tendency to have low specificity. In lung cancer early revelation of lung threat is huge. The five-year-survival-expense of lung ailment has stagnated over the most recent 30 years and is by and by at generally basically $15 \%$. Lung malignancies takes more prominent sufferers than chest development, prostate tumor and colon infection together. This is a direct result of the asymptomatic increment of this generally tumors. PC Aided Diagnosis system for acknowledgment of lung knobs (round scraped spot considerably less than $30 \mathrm{~mm}$ ) in chest radiography and CT and Computer Aided Diagnosis structures for analysis. Digital double-electricity imaging stepped forward the overall execution of CAD frameworks in chest radiography.

\subsection{CAD for Chest Radiology}

Getting a radiograph of chest has progressed toward becoming and still is the most well-known radiological exam.

A standard PA radiograph of chest (PA remains for back foremost due to this the radiation go through the influenced individual from bring down rear to frontside). The patient normally faces the onlooker: the left part of the photo demonstrates the correct lung. The lungs are radiolucent crates of air; in this manner, they show up dark inside the photo (through meeting shine sees consumed radiation) inside the lung fields, just hard figures and veins are seen [6]. The back ribs (inside the back of the patient) are noticeable all the more without a doubt; we could agree to them "flip" into the front ribs.

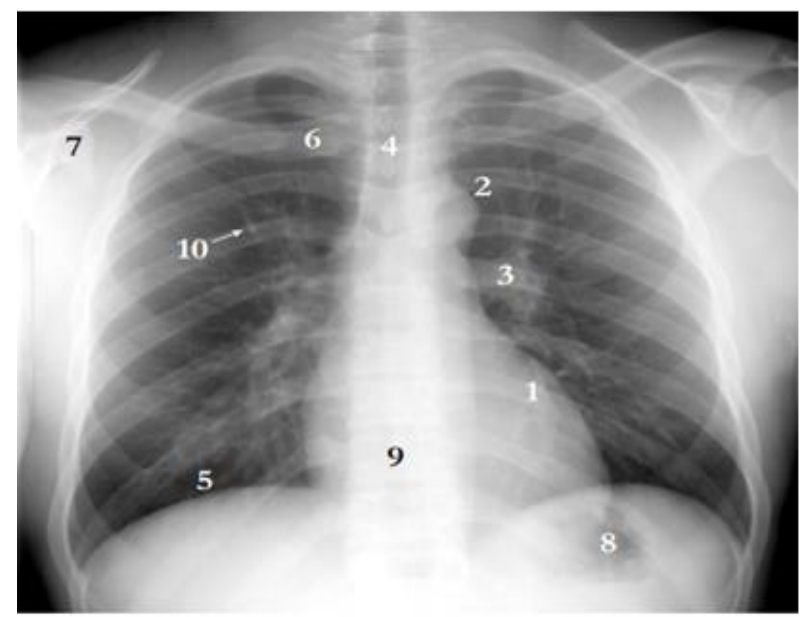

Fig. 1: Normal PA Radiograph of Chest.

It indicates a normal radiograph of chest(fig 1) with several normal figures categorized.

1) The coronary heart.

2) The aortic curve where the aorta bends.

3) The (in this example left) hilum, whereas the arteries and veins enter the lung.

4) A cloudyer vertical stripe indicates the trachea.
5) Under the lung area the diaphragm starts off evolved.

6) Clavicle

7) Shoulder blade.

8) Usually stomach gasses may be visible in the left side diaphragm.

9) If there is sufficient contrast inside the mediastinum (the field projected among the lung fields) the backbone can be visible.

10) A spherical shade like that is the shadow of the vessel that runs inside the identical course because the x-rays.

\subsection{Evaluation of CAD systems}

It offers second or alternate opinion to radiologist in diagnostic and detection techniques. Radiologists are immediate readers and selection panels. An analysis made through a radiologist the use of the output of an automated scheme for automated photograph analysis as a diagnostic useful resource [7]. With CAD (Computer Aided Diagnosis), the performance of computers does no longer need to be corresponding to or higher than that with the aid of doctors, but wishes to be equivalent to that by way of doctors (synergy). It is being utilized in experimental circumstances for more than 40 years, it usually does now not replacement the medical doctor or different skillful, but performs a role which is supporting to them [8].

\section{Materials and Methods}

Scrutinizing and diagnosing chest $\mathrm{x}$-ray pictures may be a reasonably fundamental errand for radiologists regardless, for sure, it is a mind boggling thinking issue which much of the time requires careful recognition and learning of anatomical models, physiology and pathology. Such factors augment the inconvenience of working up an enduring and automated technique for scrutinizing chest $\mathrm{X}$-shaft pictures while at the same time pondering all general thoracic infections. Coordinating, or enrollment, of pix can be characterized as finding a change that relates factors in a single photograph to their comparing focuses in some other photograph. Coordinating might be utilized for division by coordinating a sectioned reference picture to an info picture. The changed over reference picture yields a changed over division that, if the photographs are legitimately coordinated, will be an exact division of the enter picture.

A standard coordinating framework is chosen through 4 components. Reference picture (fig 2) is a urgent one since the anatomical variation between chest radiographs is huge and the picked reference picture is the form utilized amid coordinating. In the event that the reference picture isn't much similar to the info picture, up to the change searched for, coordinating will fall flat. 


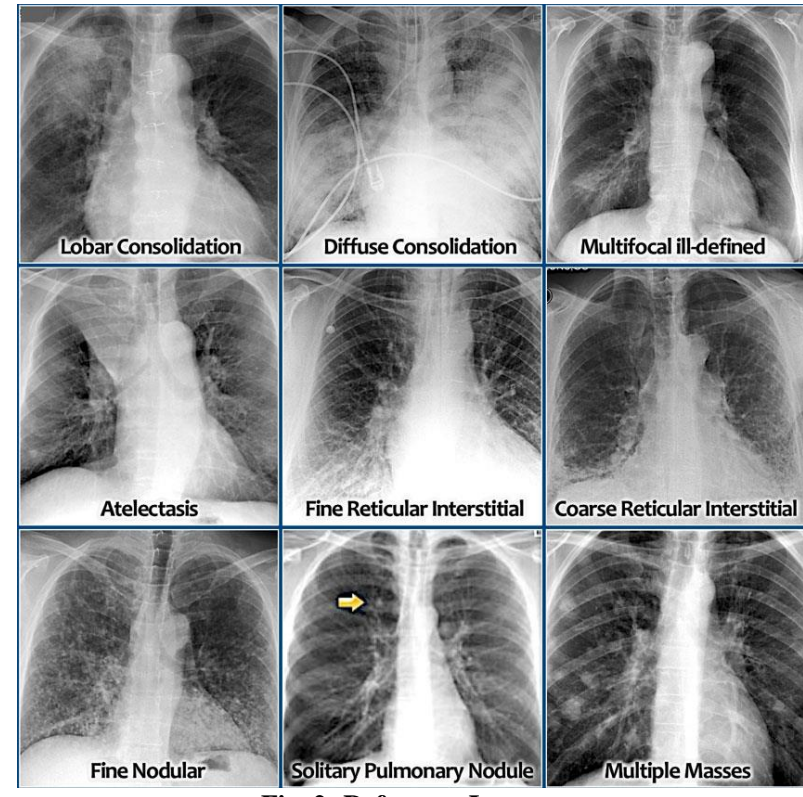

Fig. 2: Reference Images.

Second, the hunt territory is characterized through the parameter region of the permitted change. Inside the instance of lung division the utilization of reference and information pics from particular subjects, it's miles glaring that adjustments that permit anisotropic scaling are required. Third, a closeness metric is required that ought to propose how pleasantly the changed information picture and the reference picture coordinate. The last detail is the chase technique, i.e. the calculation used to choose the change which expands the closeness metric. A basic advantage of this approach is that it joins adaptation data and shape data in a verifiable way, there is no need for particular scientific styles of the articles to be fragmented, the reference picture is the model.

\subsection{Data sets used}

The datasets utilized for the present examination is $\mathrm{NIH}$ (The National Institutes of Health).NIH Clinical Centre keeps up more than 100,000 anonymized chest $\mathrm{x}$-ray pictures and their comparing information to established researchers. NIH accumulated the dataset of sweeps from in excess of 30,000 patients, incorporating numerous with cutting edge lung ailment. Fig 3, Fig 4 and Fig 5below demonstrate a portion of the chest X Rays from NIH dataset.

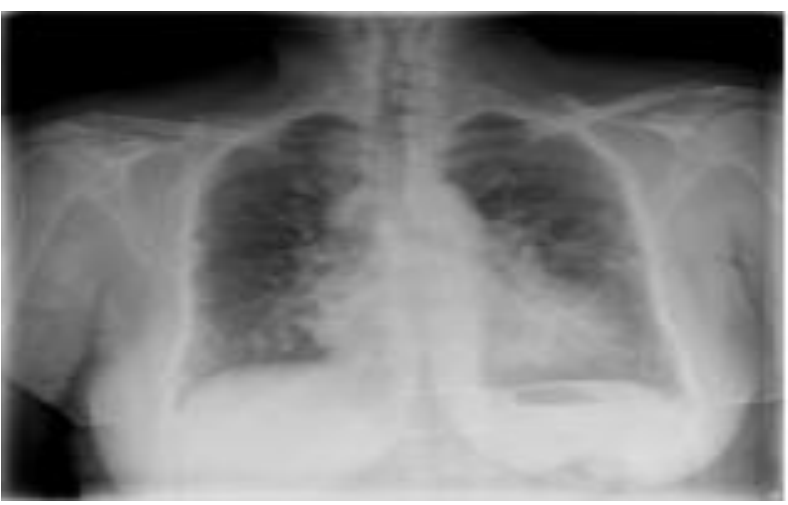

Fig. 3:.Normal Chest.

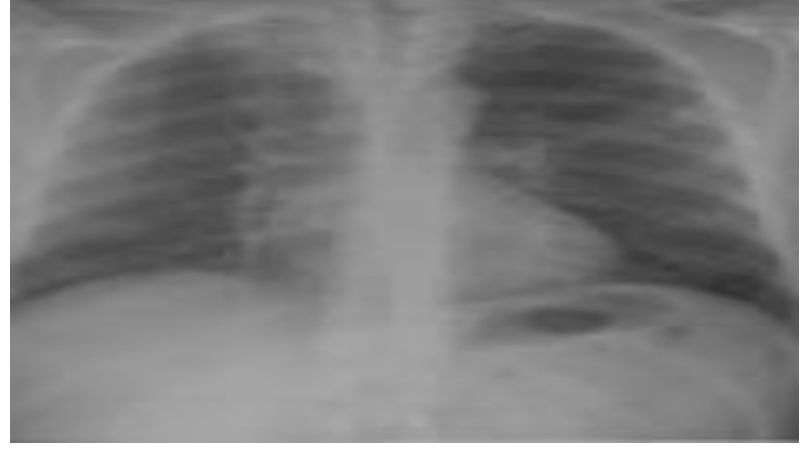

Fig. 4:.No Acute Cardio Pulmonary Process.

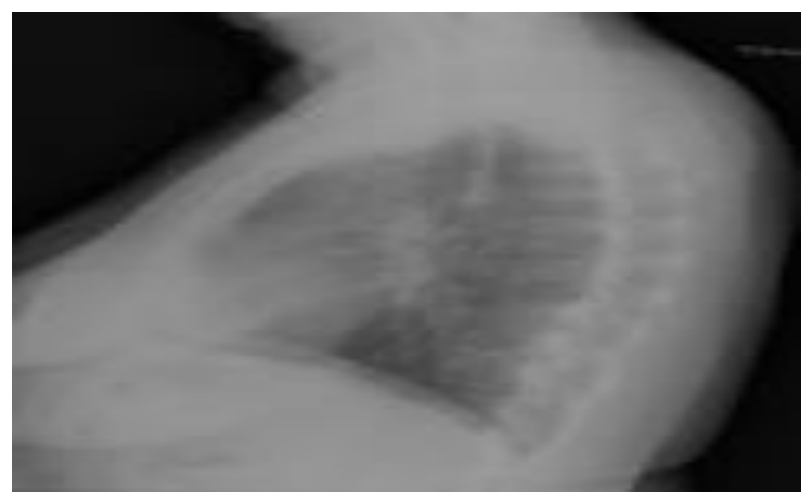

Fig. 5: Diffused Fibriosis.

\subsection{Methodology}

Computer Aided Diagnosis is essentially established on decidedly complicated sample recognition. One of the image modalities technique X-ray of medical images are scanned for careful systems. Generally some thousand pix are needed to make the algorithm to be optimized. Digital medical images data are duplicated to a CAD database in a DICOM-format which is going to be analyzed and organized in numerous steps.

Pre-processing:

Many CAD structures start by way of pre-processing the image facts. Scanned pix need to be calibrated, information ought to be resampled to a set decision, and noise elimination can be implemented. The purpose of pre-processing is to get rid of differences among information from distinct assets or obtained with specific protocols. Computer systems, being blind range crunchers, are without difficulty misled with the aid of differences that human beings can readily ignore. If a CAD device is skilled with and examined simplest on statistics from one group, as is generally the case for studies stated within the clinical literature, pre-processing may not be essential, but the results won't be generalized and won't maintain true in different settings. Reducing the antiquity of images (bugs in images), Noise reducing in images and levelling (harmonization) of quality of an image (increased contrast) for clearance of image's distinct basic conditions e.g. distinct exposure criterions, Filtering is used.

Matching:

Matching, the issue of distinguishing variations from the norm in a $\mathrm{X}$ Ray picture by thinking about the NIC dataset and the picture under investigation. The procedure thinks about the picture pixel by pixel and reports whether the picture is in casual event with the pictures in the dataset or not. In the event that coordinating is effective then the marked variation from the norm is distinguished as the danger in the recognized picture and if the coordinating is unsuccessful then a report is created indicating that the irregularity isn't recognized by the CAD procedure. Unfortunately diagram coordinating, in most of the systems[8]stays manual and work serious process so, ChestXthon mechanically trains the image to analyze abnormalities. In any case, a PC supported conclusion is required to look at two pictures of different vicinities. In this paper we depict a framework, called ChestXthon a matching procedure 
to arrange a picture as "Abnormal" or "Normal" and furthermore to indicate the threat assuming any, in the picture under investigation. If the chest photograph isn't always present inside the dataset then the algorithm is capable of trying to find a combination of photos to search for the result.

ChestXthon repeatedly trains the dataset for inclusion within the dataset as soon as the photo isn't always present in the dataset.

\subsection{Proposed algorithm}

Algorithm ChestXthon

This algorithm ChestXthon(comparison of chest $\mathrm{X}$ rays using Python) is used to compare the Radiography image with the dataset and to generate the reports disclosing the malignancy.

1) Import Image from the PIL package //This package

2) is used to work on images

3) Import openxl package //This package is used to

4) perform operations on Excel sheets

5) Input "image name" under diagnosis from command line.

6) Convert images pixel values into RGB Format shown below Image=Image. Open (FILENAME1). Convert ('RGB').

Now save the image.

pix1=image. Load ()

7) To find width and height of image

Width = int (image.size [0])

Height $=$ int $($ image.size [1])

8) Using for loop append all images pixel values into a dataset. For $\mathrm{i}$ in range (w1): // w1 is width For $\mathrm{j}$ in range (h1): // h1 is height

9) codes2.append(pix 1[i,j])

10) Whenever we are taking new image as input convert it into RGB Format and compare the pixel values with the dataset and set found="true"

11) If found="false", then there is no match with the image dataset then loop by taking a combination of pixel values of two different images in the dataset.//make the selection random by using random(dataset d1,img_new_mod)

12) If matched with multiple images display the abnormalities and store that image and abnormalities into dataset.

\subsection{Experimental setup}

General Specifications:

ChextXthon can be implemented on any Operating System with good processing capabilities and memory since the algorithm deals with a dataset of 7000 images and above, and the dataset is incremented by one after every run.

The present system is implemented in Windows Operating system: Windows 8.1 Pro 64-bit Micro Processor: AMD A10-5750M APU with Radeon(tm) HD Graphics

System Memory: 6GB

The algorithm is implemented using Python with installation of necessary packages.

\section{Experimental results}

\subsection{Screen shots obtained from python}

Screenshot 1:

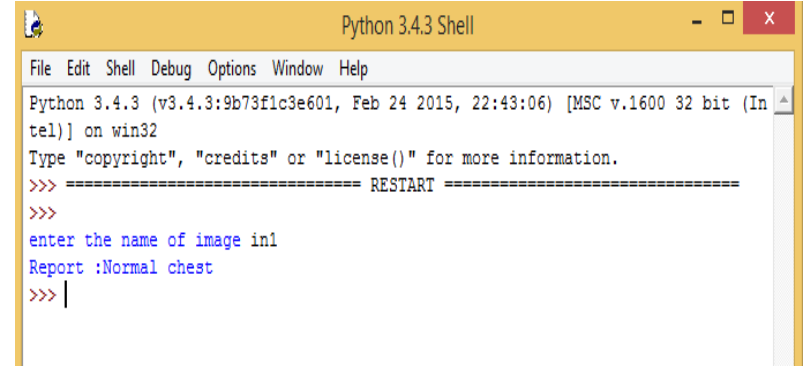

Screenshot 2:

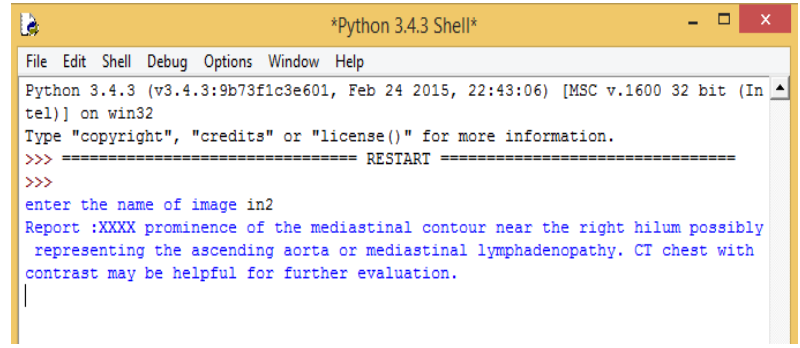

\subsection{Sample input images and the output obtained from the experiment}

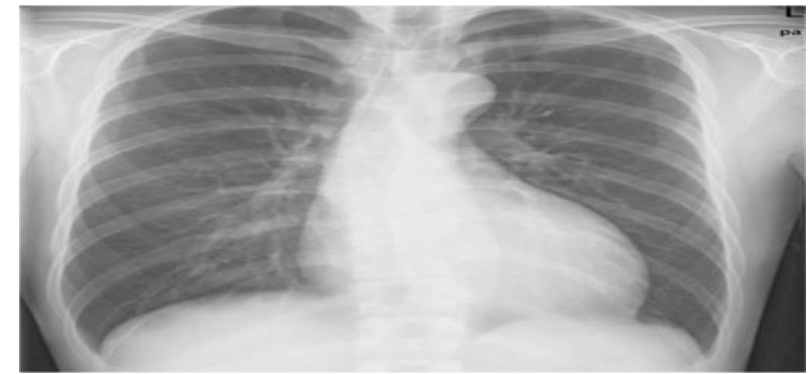

Fig. 6:.State Abnormal, and the Identified Abnormality is "Mild Cardiomegaly without Pulmonary Congestion".

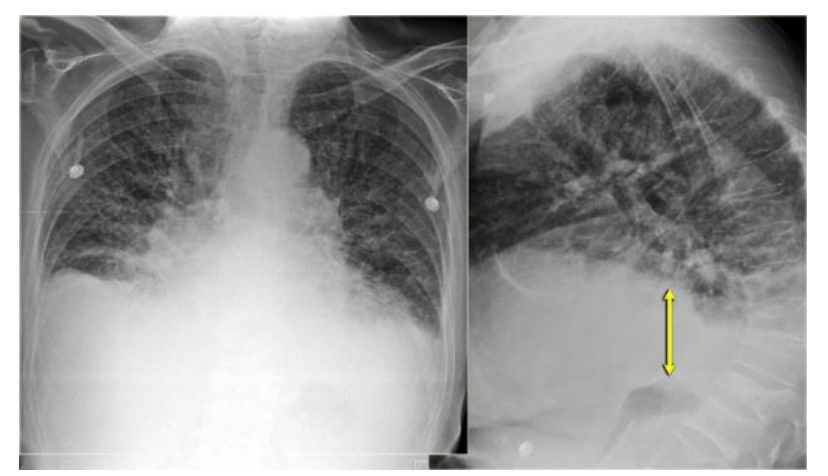

Fig. 7: State: Abnormal, and the Identified Abnormality is "Pleural Effusion". 


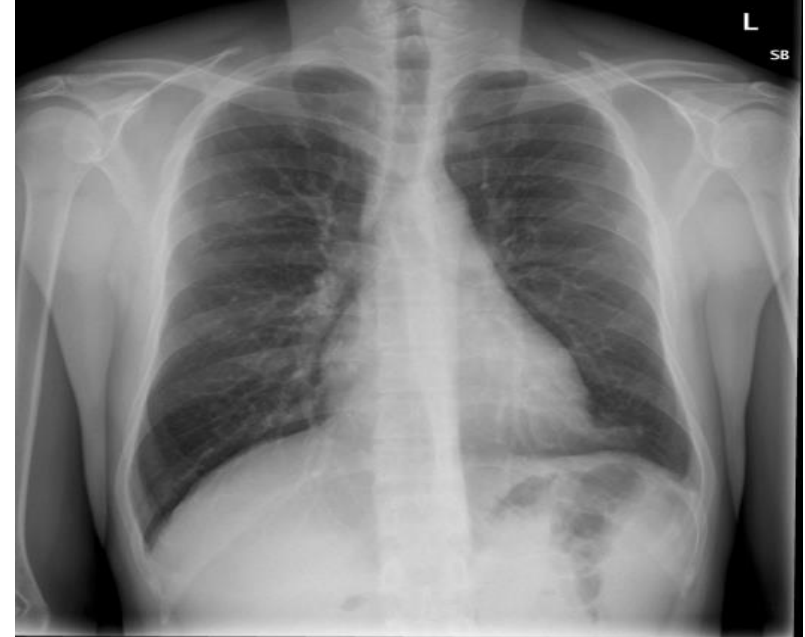

Fig. 8: State Normal, No Abnormality Identified.

\section{Discussion on results}

The algorithm ChestXthon is an astounding picture order method that goes for recognizing "normal" and "abnormal" pictures. A colossal dataset of 7000 chest pictures are being kept up in the database and the calculation experiences a thorough correlation process pixel by pixel to find the variations from the norm in ChestXRays. The calculation is composed in such a way that any new picture will be annexed to the current database. Execution of ChestXthon yields reports as shown in fig 6 , fig 7 and fig 8 .

The calculation additionally records the variations from the norm related with the strange chest pictures. ChestXthon is an incredible picture grouping apparatus to the radiologist that help them to better the procedure of finding abnormality at a brief timeframe.

\section{Conclusion and future scope}

We can presume that when we are utilizing CAD method we need to suggest radiologists that they need to take $\mathrm{x}$-rays at specific measurements as it were. Here we are utilizing coordinating system in CAD for pixel to pixel correlation of the considerable number of pictures in a dataset and show the outcomes. The algorithm can be stretched out to order pictures of any measurement without the need to resize every time another picture has showed up.

\section{References}

[1] Al-Absi HRH, Samir BB, Shaban KB, Sulaiman S. Computer aided diagnosis system based on machine learning techniques for lung cancer. In: ICCIS 2012: Proceedings of the 2012 International Conference on Computer Information Science; 2012 June 12-14; Kuala Lumpur, Malaysia. IEEE; 2012. p. 295-300. v. 1.

[2] Computer-Aided Diagnosis in Chest Radiography: A Survey Bram van Ginneken*, Bart M. terHaarRomeny, and Max A. Viergever, Member IEEE.

[3] R. Daffner, Clinical Radiology, the Essentials, 2nd ed. Baltimore MD: Williams \& Wilkins, 1999.

[4] P. Jannin, J.M. Fitzpatrick, D.J. Hawkes, X. Pennec, R. Shahidi, and M.W. Vannier. Validation of medical image processing in image-guided therapy. IEEE Transactions on Medical Imaging, 21(12):1455-1449,

2002 https://doi.org/10.1109/TMI.2002.806568

[5] K.W. Bowyer, M.H. Loew, H.S. Stiehl, and M.A. Viergever. Methodology of evaluation in medical image computing. In Rep. Dagstuhl Workshop, 2001.

[6] M. B. Stegmann, B. K. Ersbøll, and R. Larsen. FAME - a flexible appearance-modelling environment. IEEE Transactions on Medical Imaging, 22(10):1319-1331

2003 https://doi.org/10.1109/TMI.2003.817780.

[7] L. Li, Y. Zheng, M. Kallergi, and R.A. Clark. Improved method for automatic identification of lung regions on chest radiographs. Aca- demic Radiology, 8(7):629-638,

2001 https://doi.org/10.1016/S1076-6332(03)80688-8.

[8] B. van Ginneken, S. Katsuragawa, B. M. terHaarRomeny, K. Doi, and M. A. Viergever. Automatic detection of abnormalities in chest radiographs using local texture analysis. IEEE Transactions on Medical

Imaging, 21(2):139-149, 2002 . 